Centre interuniversitaire de recherche en économie quantitative

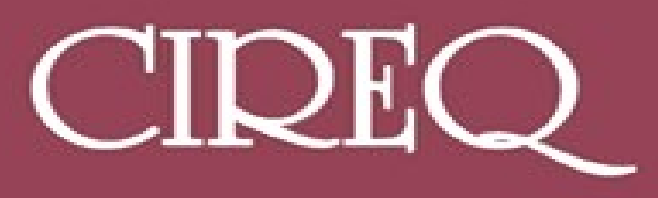

Cahier 07-2009

MEASURING ECONOMIC INSECURITY

Walter BOSSERT and Conchita D'AMBROSIO 


\title{
CIREQ
}

Le Centre interuniversitaire de recherche en économie quantitative (CIREQ) regroupe des chercheurs dans les domaines de l'économétrie, la théorie de la décision, la macroéconomie et les marchés financiers, la microéconomie appliquée et l'économie expérimentale ainsi que l'économie de l'environnement et des ressources naturelles. Ils proviennent principalement des universités de Montréal, McGill et Concordia. Le CIREQ offre un milieu dynamique de recherche en économie quantitative grâce au grand nombre d'activités qu'il organise (séminaires, ateliers, colloques) et de collaborateurs qu'il reçoit chaque année.

The Center for Interuniversity Research in Quantitative Economics (CIREQ) regroups researchers in the fields of econometrics, decision theory, macroeconomics and financial markets, applied microeconomics and experimental economics, and environmental and natural resources economics. They come mainly from the Université de Montréal, McGill University and Concordia University. CIREQ offers a dynamic environment of research in quantitative economics thanks to the large number of activities that it organizes (seminars, workshops, conferences) and to the visitors it receives every year.

\section{Cahier 07-2009}

\author{
MEASURING ECONOMIC INSECURITY
}

\author{
Walter BOSSERT and Conchita D'AMBROSIO
}

CIREQ, Université de Montréal C.P. 6128, succursale Centre-ville Montréal (Québec) H3C $3 J 7$ Canada
Université th de Montréal 
Ce cahier a également été publié par le Département de sciences économiques de I'Université de Montréal sous le numéro (2009-06).

This working paper was also published by the Department of Economics of the University of Montreal under number (2009-06).

Dépôt légal - Bibliothèque nationale du Canada, 2009, ISSN 0821-4441

Dépôt légal - Bibliothèque et Archives nationales du Québec, 2009

ISBN-13 : 978-2-89382-578-6 


\title{
Measuring Economic Insecurity*
}

\author{
WALTER Bossert \\ Department of Economics and CIREQ, University of Montreal \\ walter.bossert@umontreal.ca
}

\begin{abstract}
Conchita D'Ambrosio
Università di Milano-Bicocca, DIW Berlin and Econpubblica, Università Bocconi

conchita.dambrosio@unibocconi.it
\end{abstract}

This version: March 15, 2009

\begin{abstract}
We provide a systematic treatment of the notion of economic insecurity, assuming that an individual's sentiment of insecurity depends on the current wealth level and its variations experienced in the past. We think of wealth as a comprehensive variable encompassing anything that may help in coping with adverse occurrences. The current wealth level could also be interpreted as incorporating the individual's evaluation of future prospects. Variations in wealth experienced in the recent past are given higher weight than experiences that occurred in the more distant past. Two classes of measures are characterized with sets of plausible and intuitive axioms. Journal of Economic Literature Classification No.: D63.
\end{abstract}

Keywords: Insecurity, Wealth Distribution, Social Index Numbers.

* We thank MIUR (Prin 2007), the Fonds de Recherche sur la Société et la Culture of Québec and the Social Sciences and Humanities Research Council of Canada for financial support. For helpful comments and suggestions we thank Miguel A. Ballester and participants at the 2008 Malaga workshop on social decisions and at the 2008 Siena workshop on social choice and poverty. 


\section{Introduction}

The perception that the level of economic insecurity has risen since the 1990s and especially since the 2008 global crisis is all around us. The term 'economic insecurity' is very much used on television and radio, in the popular press, in public opinion polls and in web logs, for instance. But what exactly is meant by it? In 1998, Osberg wrote that the term 'economic insecurity' did not appear in dictionaries of economic jargon at the time (Osberg, 1998, p.22). Still, the New Palgrave Dictionary of Economics, the International Encyclopedia of Social and Behavioural Sciences and the Social Science Encyclopedia contain no entry on it. And there is more to this absence. To the best of our knowledge, there are very few papers in the economics literature which have dealt with defining and measuring it. "Economists have written a great deal about risk, but very little about 'insecurity'," according to Osberg (1998, p.22). He continues by saying that "[A] definition of economic insecurity which reflects the common usage meaning of the term insecure might be: "the anxiety produced by the lack of economic safety"." (Osberg, 1998, p.23). According to the United Nations Department of Economic and Social Affairs (2008, p.vi), "It is not easy to give a precise meaning to the term economic insecurity. Partly because it often draws on comparisons with past experiences and practices, which have a tendency to be viewed through rose-tinted lenses, and also because security has a large subjective or psychological component linked to feelings of anxiety and safety, which draw heavily on personal circumstances. Still in general terms economic insecurity arises from the expo-

sure of individuals, communities and countries to adverse events, and from their inability to cope with and recover from the costly consequences of those events." Jacobs (2007) suggests that "Economic insecurity is perhaps best understood as the intersection between "perceived" and "actual" downside risk." The Berkeley Center on Health, Economic and Family Security is working on a 'Rockefeller Economic Security Index' but no results are currently available.

If we had to summarize in one sentence what we found based on common threads of the above quotations, we could say that economic insecurity is the anxiety produced by the exposure to adverse events and the inability to recover from them. Past, present and future are all involved. We are insecure about the future, since the future is all that matters for generating anxiety to an individual - we know where we are today and the past cannot be changed. The resources we have today are important: the wealthier we are, the bigger the buffer stock we can rely on in case of an adverse future event. Our past experiences play a role in shaping our self-confidence on how well we can do in case 
of an adverse event. We remember gains and losses in our resources over time. Of course, the more recent these variations are, the more vivid our memories.

The aim of the paper is to formalize this idea by providing a systematic treatment of the notion of economic insecurity. We assume that an individual's sentiment of insecurity today depends on the current wealth level and variations in wealth experienced in the past. We think of wealth as a comprehensive variable that encompasses anything that may help an individual in coping with adverse occurrences. The current wealth level could also be interpreted as the present value of all expected changes in future wealth. This value will take into account the individual's evaluation of future prospects. First, we propose a set of three basic properties that we think a measure of economic insecurity should possess. We then move on to more specific classes of measures that, in spirit, are related to the generalized Gini social welfare functions. According to these measures, insecurity is given by the current wealth level multiplied by minus one plus weighted sums of the wealth gains (losses) experienced in the past. Two sequences of coefficients are employed - one applied to gains, one applied to losses. The coefficients are such that recent experiences are given higher weight than experiences that have occurred in the more distant past. A subclass of these measures is obtained by giving higher weights to the absolute values of past losses than to those of past gains, reflecting an attitude analogous to risk aversion in models of individual decision making under uncertainty. Both the class of two-sequences Ginis and the latter subclass are characterized with sets of plausible and intuitive axioms.

The paper is organized as follows. The next section presents the formal framework and the properties we think a measure of economic insecurity should possess. In section 3 we characterize some measures of the phenomenon. Section 4 concludes.

\section{Wealth Streams and Individual Insecurity}

For any $T \in \mathbb{N}_{0}$, let $\mathbb{R}^{(T)}$ be the $(T+1)$-dimensional Euclidean space with components labeled $(-T, \ldots, 0)$. Zero is interpreted as the current period and $T$ is the number of past periods taken into consideration (which may vary depending on the data available). A measure of individual insecurity is a sequence of functions $V=\left\langle V^{T}\right\rangle_{T \in \mathbb{N}_{0}}$, where, for each $T \in \mathbb{N}_{0}, V^{T}: \mathbb{R}^{(T)} \rightarrow \mathbb{R}$. The measure assigns a degree of insecurity to each individual wealth stream $w \in \bigcup_{T \in \mathbb{N}_{0}} \mathbb{R}^{(T)}$. We allow (net) wealth to be negative. The wealth stream $w=(1,3,3,-1,0,2) \in \mathbb{R}^{(5)}$ is illustrated in Figure 1. 


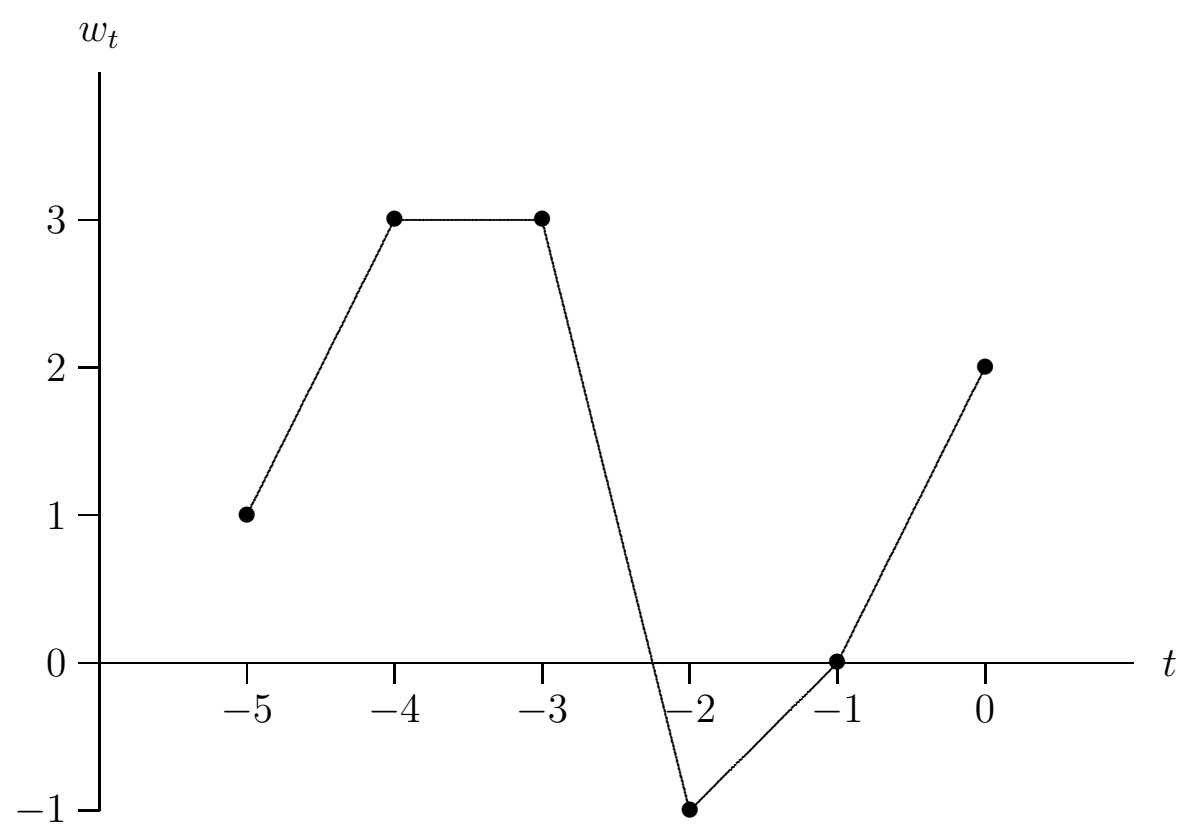

Figure 1: The Wealth Stream $w=(1,3,3,-1,0,2)$.

It is possible to think of $w_{0}$ as encompassing not only the current wealth level of the individual but also her or his assessment of his or her (uncertain) future wealth levels. We do not want to commit to a specific method of forming expectations about the future which is why we choose this general formulation. For convenience, we will continue to refer to $w_{0}$ as current wealth, keeping in mind that this figure may include expectations regarding future wealth levels.

We want to design a class of measures the members of which capture the dependence of a sentiment of insecurity on past wealth movements in addition to today's wealth level. Loosely speaking, the basic hypothesis is that insecurity increases (decreases), ceteris paribus, with decreases (increases) of the wealth level experienced in the past with higher weight given to the recent past. Thus, we propose to use the following three axioms as the defining properties of an individual measure of economic insecurity, provided that the observed variable is individual net wealth.

Single-period monotonicity. $V^{0}$ is a decreasing function of $w_{0}$.

The interpretation of single-period monotonicity is straightforward and intuitive: in the absence of any information regarding past wealth levels, insecurity is inversely related to the current wealth level.

Difference monotonicity. For all $T \in \mathbb{N}$, for all $w \in \mathbb{R}^{(T-1)}$ and for all $\gamma \in \mathbb{R}$,

$$
V^{T}\left(w_{-(T-1)}+\gamma, w\right) \geq V^{T-1}(w) \Leftrightarrow \gamma \geq 0 .
$$


Difference monotonicity requires a decrease in insecurity as a consequence of the ceterisparibus addition of another period $-T$ which introduces a gain between periods $-T$ and $-(T-1)$, thus allowing past gains to work against insecurity. Analogously, the measure of insecurity is assumed to increase if a period $-T$ is added in a way such that wealth decreases, ceteris paribus, when moving from $-T$ to $-(T-1)$. Finally, if the addition of period $-T$ involves a wealth level identical to that of period $-(T-1)$, insecurity is unchanged. This is a monotonicity requirement that appears to be essential in capturing the notion of increased (decreased, unchanged, respectively) insecurity as a response to additional losses (additional gains, no changes, respectively) in wealth levels experienced in the past. Note that the axiom does not imply that gains and losses have to be treated symmetrically; it is possible, for instance, that adding a gain of a certain magnitude, ceteris paribus, decreases insecurity by less than a loss of the same magnitude increases insecurity. We will return to this issue in more detail in the next section. The axiom is illustrated in Figure 2. Starting from $w^{\prime}=(3,3,-1,0,2) \in \mathbb{R}^{(4)}$, an additional period -5 is added to arrive at the stream $w=(1,3,3,-1,0,2) \in \mathbb{R}^{(5)}$. The move from period -5 to period -4 involves a gain in net wealth and, thus, difference monotonicity demands that $V^{5}(w)<V^{4}\left(w^{\prime}\right)$.

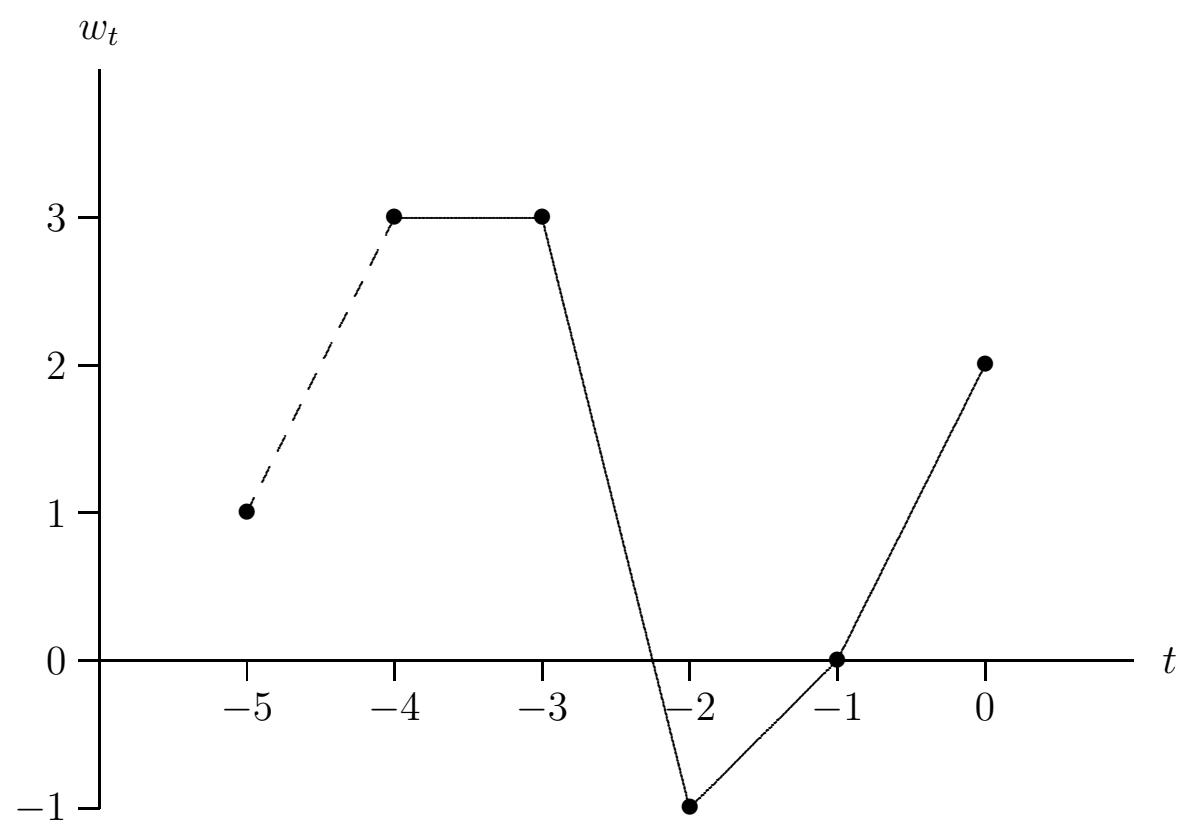

Figure 2: Difference Monotonicity. 
Proximity property. For all $T \in \mathbb{N} \backslash\{1\}$, for all $w \in \mathbb{R}^{(T)}$ and for all $\tau \in\{1, \ldots, T-1\}$,

$$
\begin{array}{cc} 
& V^{T}\left(w_{-T}, \ldots, w_{-(\tau+1)}, w_{-(\tau+1)}, w_{-(\tau-1)}, \ldots, w_{0}\right) \geq \\
& V^{T}\left(w_{-T}, \ldots, w_{-(\tau+1)}, w_{-(\tau-1)}, w_{-(\tau-1)}, \ldots, w_{0}\right) \\
\Leftrightarrow \quad & w_{-(\tau+1)} \geq w_{-(\tau-1)} .
\end{array}
$$

The proximity property ensures that a gain (loss) of a given magnitude reduces (increases) insecurity, ceteris paribus, to a higher extent the closer to the present this gain (loss) occurs. That is, changes in wealth from one period to the next have a more severe impact the closer they are to the present period. Figure 3 illustrates the axiom. Comparing the streams $w=(1,3,3,-1,0,2) \in \mathbb{R}^{(5)}$ and $w^{\prime}=(1,3,-1,-1,0,2) \in \mathbb{R}^{(5)}$, we see that $w^{\prime}$ can be obtained from $w$ by shifting the drop from 3 to -1 one period further into the past. According to the proximity property, the earlier loss affects the current sentiment of insecurity to a lesser extent than the original one and, thus, insecurity in $w^{\prime}$ is less that insecurity in $w$.

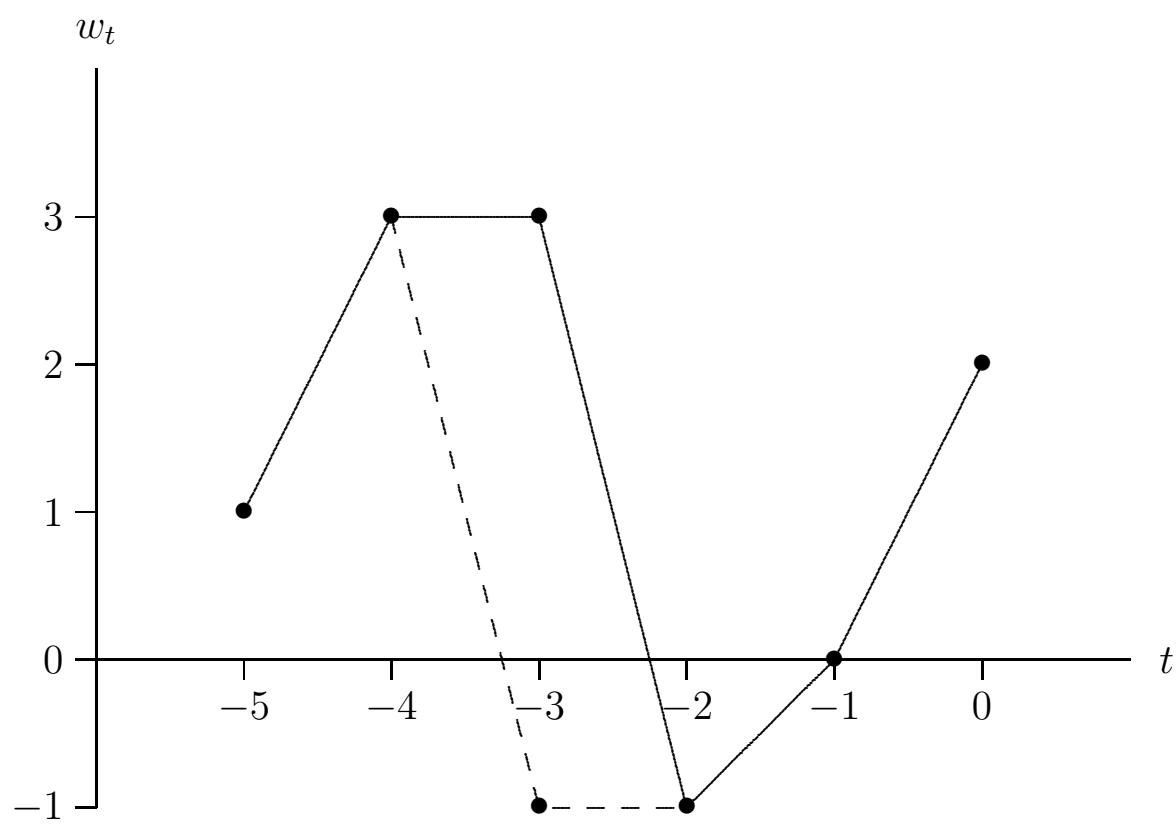

Figure 3: Proximity Property.

We suggest to use the above three axioms as the fundamental properties of a measure of individual insecurity. This is parallel to the definition of an inequality measure as an S-convex function of individual incomes. 


\section{Two-Sequences Gini Measures}

We now characterize a specific class of measures that are inspired by the single-series Gini measures of inequality. They constitute special cases of the generalized Ginis where the sequence of coefficients is the same for all population sizes. See, for instance, Donaldson and Weymark (1980), Weymark (1981) and Bossert (1990) for a discussion of the generalized and the single-series Ginis. Zank (2007) includes the generalized Ginis in his analysis of welfare functions with a reference income. Our characterization bears some resemblance to that of Bossert (1990) but it differs from this earlier contribution in that we work with a different domain and axioms that are suited to the environment discussed here.

The class of two-sequences Gini measures involves two sequences of parameters - one the members of which are applied to past losses in wealth, one that is used for those period pairs in which there are gains. The sequences need not be the same but, within each sequence, some natural restrictions apply. Let $\alpha=\left\langle\alpha_{-t}\right\rangle_{t \in \mathbb{N}}$ and $\beta=\left\langle\beta_{-t}\right\rangle_{t \in \mathbb{N}}$ be two sequences of parameters such that

$$
\left[\alpha_{-t}>\alpha_{-(t+1)}>0 \text { and } \quad \beta_{-t}>\beta_{-(t+1)}>0\right] \quad \text { for all } t \in \mathbb{N} \text {. }
$$

The set of all sequences $\alpha$ such that $\alpha_{-t}>\alpha_{-(t+1)}>0$ for all $t \in \mathbb{N}$ is denoted by $\mathcal{C}$. $\mathcal{C}^{2}$ is the Cartesian product of $\mathcal{C}$ with itself, that is, $\mathcal{C}^{2}$ is the set of all pairs of sequences satisfying (1). The two-sequences Gini measure of insecurity corresponding to a pair of sequences $(\alpha, \beta) \in \mathcal{C}^{2}, V_{(\alpha, \beta)}=\left\langle V_{(\alpha, \beta)}^{T}\right\rangle_{T \in \mathbb{N}_{0}}$, is defined by letting, for all $T \in \mathbb{N}_{0}$ and for all $w=\left(w_{-T}, \ldots, w_{0}\right) \in \mathbb{R}^{(T)}$,

$$
V_{(\alpha, \beta)}^{T}(w)=\sum_{\substack{t \in\{1, \ldots, T\}: \\ w_{-t}>w_{-}(t-1)}} \alpha_{-t}\left(w_{-t}-w_{-(t-1)}\right)+\sum_{\substack{t \in\{1, \ldots, T\}: \\ w_{-t}<w_{-}(t-1)}} \beta_{-t}\left(w_{-t}-w_{-(t-1)}\right)-w_{0}
$$

As an illustration of the two-sequences Gini measures, consider Figure 4. We use the parameter values $\alpha_{-1}=1$ and $\beta_{-1}=1 / 2$ and depict the level sets of $V_{(1,1 / 2)}^{1}(w)$ corresponding to the levels 1 (the piecewise linear set farthest to the northwest), 0 (the middle piecewise linear set) and -1 (the piecewise linear set farthest to the southeast). 


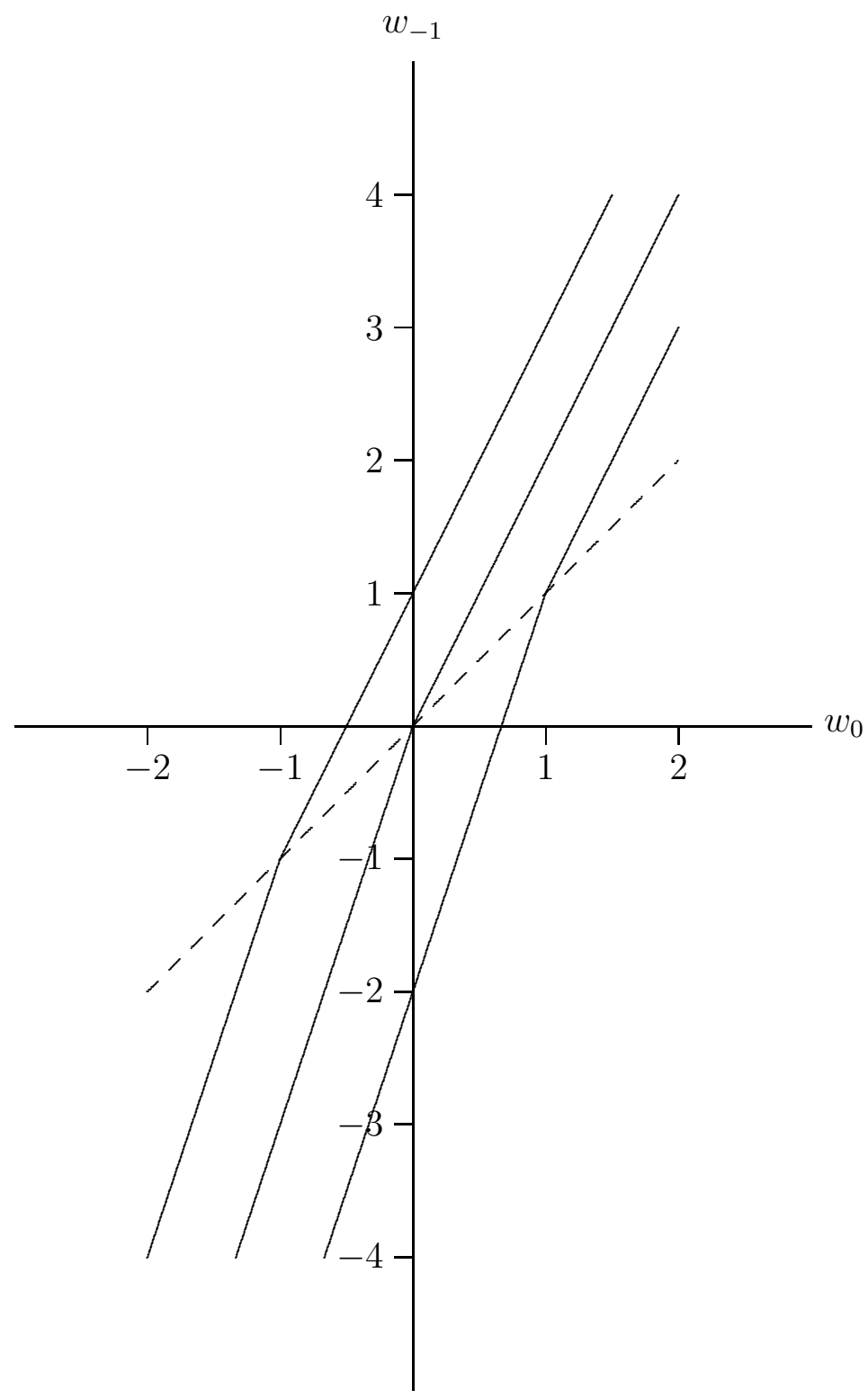

Figure 4: Level Sets of $V_{(1,1 / 2)}^{1}(w)$ for Values 1, 0 and -1 .

In addition to the defining properties of an individual measure of insecurity introduced in the previous section, the following axioms are used to characterize the class of twosequences Gini measures of individual insecurity. For $r \in \mathbb{N}$, we use $\mathbf{1}_{r}$ to denote the vector consisting of $r$ ones.

Homogeneity. For all $T \in \mathbb{N}_{0}$, for all $w \in \mathbb{R}^{(T)}$ and for all $\lambda \in \mathbb{R}_{++}$,

$$
V^{T}(\lambda w)=\lambda V^{T}(w)
$$


Homogeneity is a standard requirement that demands insecurity to be measured by means of a ratio scale.

Translatability. For all $T \in \mathbb{N}_{0}$, for all $w \in \mathbb{R}^{(T)}$ and for all $\delta \in \mathbb{R}$,

$$
V^{T}\left(w+\delta \mathbf{1}_{T+1}\right)=V^{T}(w)-\delta
$$

Translatability differs from the usual translation-scale property in that the value of $\delta$ is subtracted from the level of insecurity when $\delta$ is added to the wealth level in each period. This is a consequence of the inverse relationship between wealth and insecurity.

Homogeneity and translatability together imply a strengthening of single-period monotonicity, as stated in the following lemma.

Lemma 1. If a measure of individual insecurity $V$ satisfies homogeneity and translatability, then

$$
V^{0}\left(w_{0}\right)=-w_{0} \quad \text { for all } w_{0} \in \mathbb{R}
$$

Proof. Setting $T=0$ and $w_{0}=0$, homogeneity implies

$$
V^{0}(0)=V^{0}(\lambda \cdot 0)=\lambda V^{0}(0) \quad \text { for all } \lambda \in \mathbb{R}_{++}
$$

and, substituting any $\lambda \neq 1$, it follows that

$$
V^{0}(0)=0
$$

Setting $T=0$ and $\delta=-w_{0}$ in the definition of translatability and using (3), we obtain

$$
V^{0}(0)=V^{0}\left(w_{0}+\left(-w_{0}\right)\right)=V^{0}\left(w_{0}\right)+w_{0}=0 \quad \text { for all } w_{0} \in \mathbb{R}
$$

Clearly, the last equality in (4) is equivalent to (2).

Note that the full force of homogeneity and translatability is not needed for the above lemma; as is evident from the proof, it is sufficient to use the respective properties that are obtained by restricting the scopes of the axioms to the cases in which $T=0$.

Temporal aggregation property. For all $T \in \mathbb{N} \backslash\{1\}$, there exists a function $\Phi^{T}: \mathbb{R}^{2} \rightarrow$ $\mathbb{R}$ such that, for all $w \in \mathbb{R}^{(T)}$,

$$
V^{T}(w)=\Phi^{T}\left(w_{-T}-w_{-(T-1)}, V^{T-1}\left(w_{-(T-1)}, \ldots, w_{0}\right)\right)
$$


The temporal aggregation property is a separability condition that allows the measure of insecurity to be calculated by recursively moving back from the current period to the earliest relevant period where, in the step involving period $-t$, the part of insecurity that takes into consideration all periods from $-t$ to the current period is obtained as an aggregate of the insecurity resulting from considering periods $-(t-1)$ to period zero only and the change experienced in the wealth level between periods $-t$ and $-(t-1)$; see Blackorby, Primont and Russell (1978) for a detailed discussion of various recursivity properties.

If added to the basic requirements of the previous section (except for single-period monotonicity which is redundant; see Lemma 1), these axioms characterize the class of two-parameter Gini measures of insecurity. We obtain

Theorem 1. A measure of individual insecurity $V$ satisfies difference monotonicity, the proximity property, homogeneity, translatability and the temporal aggregation property if and only if there exists $(\alpha, \beta) \in \mathcal{C}^{2}$ such that $V=V_{(\alpha, \beta)}$.

Proof. 'If.' Let $(\alpha, \beta) \in \mathcal{C}^{2}$. That $V_{(\alpha, \beta)}$ satisfies homogeneity and translatability is immediate. Difference monotonicity follows from the positivity of the coefficients $\alpha_{-t}$ and $\beta_{-t}$; see the definition of $\mathcal{C}$. The proximity property is satisfied because of the inequalities that apply to the sequences of parameters; see, again, the definition of $\mathcal{C}$. To see that the temporal aggregation property is satisfied, define, for all $T \in \mathbb{N} \backslash\{1\}$, the function $\Phi^{T}: \mathbb{R}^{2} \rightarrow \mathbb{R}$ by letting, for all $(x, y) \in \mathbb{R}^{2}$,

$$
\Phi^{T}(x, y)= \begin{cases}\alpha_{-T} x+y & \text { if } x>0 \\ y & \text { if } x=0 \\ \beta_{-T} x+y & \text { if } x<0 .\end{cases}
$$

'Only if.' Suppose $V$ satisfies the required axioms. We prove the relevant implication by inductively constructing a pair of sequences $(\alpha, \beta) \in \mathcal{C}^{2}$ such that

$$
V^{T}(w)=\sum_{\substack{t \in\{1, \ldots, T\}: \\ w_{-t}>w_{-}(t-1)}} \alpha_{-t}\left(w_{-t}-w_{-(t-1)}\right)+\sum_{\substack{t \in\{1, \ldots, T\}: \\ w_{-t} w_{-(t-1)}}} \beta_{-t}\left(w_{-t}-w_{-(t-1)}\right)-w_{0}
$$

for all $T \in \mathbb{N}_{0}$ and for all $w \in \mathbb{R}^{(T)}$.

If $T=0$, (5) is satisfied for all $w=\left(w_{0}\right) \in \mathbb{R}^{(0)}$ (trivially, for any pair $(\alpha, \beta) \in \mathcal{C}^{2}$ and, in particular, for the pair of sequences to be constructed below) because of (2).

Now let $T=1$. 
If $w \in \mathbb{R}^{(1)}$ is such that $w_{-1}=w_{0}$, monotonicity and (2) together imply

$$
V^{1}(w)=V^{0}(w)=-w_{0}
$$

If $w$ is such that $w_{-1}>w_{0}$, translatability with $\delta=-w_{0}$ implies

$$
V^{1}\left(w_{-1}-w_{0}, 0\right)=V^{1}\left(w_{-1}-w_{0}, w_{0}-w_{0}\right)=V^{1}\left(w_{-1}, w_{0}\right)+w_{0}=V^{1}(w)+w_{0}
$$

and, therefore,

$$
V^{1}(w)=V^{1}\left(w_{-1}-w_{0}, 0\right)-w_{0} .
$$

Applying homogeneity with $\lambda=w_{-1}-w_{0}>0$, it follows that

$$
V^{1}\left(w_{-1}-w_{0}, 0\right)=V^{1}\left(\left(w_{-1}-w_{0}\right) \cdot 1,\left(w_{-1}-w_{0}\right) \cdot 0\right)=\left(w_{-1}-w_{0}\right) V^{1}(1,0)
$$

and, together with (7),

$$
V^{1}(w)=\alpha_{-1}\left(w_{-1}-w_{0}\right)-w_{0}
$$

where $\alpha_{-1}=V^{1}(1,0)$. By difference monotonicity, $\alpha_{-1}>0$.

If $w$ is such that $w_{-1}<w_{0}$, translatability with $\delta=-w_{0}$ implies

$$
V^{1}\left(w_{-1}-w_{0}, 0\right)=V^{1}\left(w_{-1}-w_{0}, w_{0}-w_{0}\right)=V^{1}\left(w_{-1}, w_{0}\right)+w_{0}=V^{1}(w)+w_{0}
$$

and, therefore,

$$
V^{1}(w)=V^{1}\left(w_{-1}-w_{0}, 0\right)-w_{0} .
$$

Applying homogeneity with $\lambda=-\left(w_{-1}-w_{0}\right)>0$, it follows that

$$
V^{1}\left(\left(w_{-1}-w_{0}\right), 0\right)=V^{1}\left(-\left(w_{-1}-w_{0}\right) \cdot(-1),-\left(w_{-1}-w_{0}\right) \cdot 0\right)=-\left(w_{-1}-w_{0}\right) V^{1}(-1,0)
$$

and, together with (9),

$$
V^{1}(w)=\beta_{-1}\left(w_{-1}-w_{0}\right)-w_{0}
$$

where $\beta_{-1}=-V^{1}(-1,0)$. By difference monotonicity, $\beta_{-1}>0$.

Combining (6), (8) and (10), we obtain

$$
V^{1}(w)=\sum_{\substack{t \in\{1\}: \\ w_{-t}>w_{-}(t-1)}} \alpha_{-t}\left(w_{-t}-w_{-(t-1)}\right)+\sum_{\substack{t \in\{1\}: \\ w_{-t}<w_{-(t-1)}}} \beta_{-t}\left(w_{-t}-w_{-(t-1)}\right)-w_{0}
$$

for all $w \in \mathbb{R}^{(1)}$.

Now suppose that $T \in \mathbb{N} \backslash\{1\}$ and

$$
V^{T-1}(w)=\sum_{\substack{t \in\{1, \ldots, T-1\}: \\ w_{-t}>w_{-(t-1)}}} \alpha_{-t}\left(w_{-t}-w_{-(t-1)}\right)+\sum_{\substack{t \in\{1, \ldots, T-1\}: \\ w_{-t}<w_{-}(t-1)}} \beta_{-t}\left(w_{-t}-w_{-(t-1)}\right)-w_{0}
$$


for all $w \in \mathbb{R}^{(T-1)}$ where $\left(\alpha_{-(T-1)}, \ldots, \alpha_{-1}\right)$ and $\left(\beta_{-(T-1)}, \ldots, \beta_{-1}\right)$ are such that $\alpha_{-1}>$ $\ldots>0$ and $\beta_{-1}>\ldots>0$. We have to show that there exists $\left(\alpha_{-T}, \beta_{-T}\right)$ such that

$$
\alpha_{-1}>\ldots>\alpha_{-T}>0 \text { and } \beta_{-1}>\ldots>\beta_{-T}>0
$$

and

$$
V^{T}(w)=\sum_{\substack{t \in\{1, \ldots, T\}: \\
w_{-t}>w_{-}(t-1)}} \alpha_{-t}\left(w_{-t}-w_{-(t-1)}\right)+\sum_{\begin{array}{c}
t \in\{1, \ldots, T\}: \\
w_{-t}<w_{-}(t-1)
\end{array}} \beta_{-t}\left(w_{-t}-w_{-(t-1)}\right)-w_{0}
$$

for all $w \in \mathbb{R}^{(T)}$.

Together with (11), the temporal aggregation property implies the existence of a function $\Phi^{T}: \mathbb{R}^{2} \rightarrow \mathbb{R}$ such that

$$
\begin{aligned}
V^{T}(w)= & \Phi^{T}\left(w_{-T}-w_{-(T-1)}, V^{T-1}\left(w_{-(T-1)}, \ldots, w_{0}\right)\right) \\
= & \Phi^{T}\left(w_{-T}-w_{-(T-1)}, \sum_{\substack{t \in\{1, \ldots, T-1\}: \\
w_{-t}>w_{-(t-1)}}} \alpha_{-t}\left(w_{-t}-w_{-(t-1)}\right)\right. \\
& \left.+\sum_{\substack{t \in\{1, \ldots, T-1\}: \\
w_{-t}<w_{-(t-1)}}} \beta_{-t}\left(w_{-t}-w_{-(t-1)}\right)-w_{0}\right)
\end{aligned}
$$

for all $w \in \mathbb{R}^{(T)}$.

First, consider $w \in \mathbb{R}^{(T)}$ such that $w_{-T}=w_{-(T-1)}$. Difference monotonicity and (11) together imply

$$
\begin{aligned}
V^{T}(w) & =V^{T-1}\left(w_{-(T-1)}, \ldots, w_{0}\right) \\
& =\sum_{\substack{t \in\{1, \ldots, T-1\}: \\
w_{-t}>w_{-}(t-1)}} \alpha_{-t}\left(w_{-t}-w_{-(t-1)}\right)+\sum_{\substack{t \in\{1, \ldots, T-1\}: \\
w_{-t}<w_{-}(t-1)}} \beta_{-t}\left(w_{-t}-w_{-(t-1)}\right)-w_{0}
\end{aligned}
$$

and it follows that

$$
\Phi^{T}(0, y)=y \quad \text { for all } y \in \mathbb{R}^{2} .
$$

Now consider the case in which $w$ is such that $w_{-T}>w_{-(T-1)}$. Homogeneity implies that $\Phi^{T}$ satisfies

$$
\Phi^{T}(\lambda x, \lambda y)=\lambda \Phi^{T}(x, y) \quad \text { for all } \lambda, x \in \mathbb{R}_{++} \text {and for all } y \in \mathbb{R}
$$

and translatability implies

$$
\Phi^{T}(x, y-\delta)=\Phi^{T}(x, y)-\delta \quad \text { for all } x \in \mathbb{R}_{++} \text {and for all } \delta, y \in \mathbb{R}
$$


Letting $\delta=y,(17)$ implies $\Phi^{T}(x, 0)=\Phi^{T}(x, y)-y$ and, thus,

$$
\Phi^{T}(x, y)=\Phi^{T}(x, 0)+y \quad \text { for all } x \in \mathbb{R}_{++} \text {and for all } y \in \mathbb{R} .
$$

Letting $\lambda=x>0,(16)$ implies

$$
\Phi^{T}(x, 0)=\Phi^{T}(x \cdot 1, x \cdot 0)=x \Phi^{T}(1,0) \quad \text { for all } x \in \mathbb{R}_{++}
$$

and, together with (18), we obtain

$$
\Phi^{T}(x, y)=\alpha_{-T} x+y \quad \text { for all } x \in \mathbb{R}_{++} \text {and for all } y \in \mathbb{R}
$$

with $\alpha_{-T}=\Phi^{T}(1,0)$. By difference monotonicity, $\alpha_{-T}>0$ and by the proximity property, $\alpha_{-T}<\alpha_{-(T-1)}$ and, thus,

$$
\alpha_{-1}>\ldots>\alpha_{-T}>0
$$

Finally, suppose $w$ is such that $w_{-T}<w_{-(T-1)}$. Homogeneity implies that $\Phi^{T}$ satisfies

$$
\Phi^{T}(\lambda x, \lambda y)=\lambda \Phi^{T}(x, y) \quad \text { for all } \lambda \in \mathbb{R}_{++} \text {, for all } x \in \mathbb{R}_{--} \text {and for all } y \in \mathbb{R}
$$

and translatability implies

$$
\Phi^{T}(x, y-\delta)=\Phi^{T}(x, y)-\delta \quad \text { for all } x \in \mathbb{R}_{--} \text {and for all } \delta, y \in \mathbb{R} \text {. }
$$

Letting $\delta=y,(22)$ implies $\Phi^{T}(x, 0)=\Phi^{T}(x, y)-y$ and, thus,

$$
\Phi^{T}(x, y)=\Phi^{T}(x, 0)+y \quad \text { for all } x \in \mathbb{R}_{--} \text {and for all } y \in \mathbb{R} .
$$

Letting $\lambda=-x>0,(21)$ implies

$$
\Phi^{T}(x, 0)=\Phi^{T}(-x \cdot(-1),-x \cdot 0)=-x \Phi^{T}(-1,0) \quad \text { for all } x \in \mathbb{R}_{--}
$$

and, together with (23), we obtain

$$
\Phi^{T}(x, y)=\beta_{-T} x+y \quad \text { for all } x \in \mathbb{R}_{--} \text {and for all } y \in \mathbb{R}
$$

with $\beta_{-T}=-\Phi^{T}(-1,0)$. By difference monotonicity, $\beta_{-T}>0$ and by the proximity property, $\beta_{-T}<\beta_{-(T-1)}$ and, thus,

$$
\beta_{-1}>\ldots>\beta_{-T}>0 \text {. }
$$

Combining (15), (19) and (24), it follows that

$$
\Phi^{T}(x, y)= \begin{cases}\alpha_{-T} x+y & \text { if } x>0 \\ y & \text { if } x=0 \\ \beta_{-T} x+y & \text { if } x<0\end{cases}
$$


for all $(x, y) \in \mathbb{R}^{2}$. Substituting back into (14), we obtain (13) for all $w \in \mathbb{R}^{(T)}$. Because $\alpha_{-1}>0$ and $\beta_{-1}>0$ and, moreover, (20) and (25) are satisfied for all $T \in \mathbb{N} \backslash\{1\}$, the pair of sequences $(\alpha, \beta)$ thus constructed satisfies (12) and therefore is in $\mathcal{C}^{2}$ as required.

The above theorem does not impose any restrictions on the relationship between the sequences $\alpha$ and $\beta$. A plausible assumption appears to be the requirement that ceteris paribus losses of a certain magnitude have at least as strong an impact on insecurity as ceteris paribus gains of the same magnitude. For instance, a loss in wealth caused by a financial-sector crisis is likely to increase one's sentiment of insecurity to an extent that is, in absolute value, at least as large as the effect of a gain of the same magnitude on insecurity. Analogously, the effect of a lost health benefit may very well increase one's sense of insecurity by an amount that is at least as high as the decrease in insecurity resulting from gaining this benefit. This assumption is captured in the following axiom.

Weak loss priority. For all $T \in \mathbb{N}$, for all $w \in \mathbb{R}^{(T-1)}$ and for all $\gamma \in \mathbb{R}_{++}$,

$$
V^{T}\left(w_{-(T-1)}+\gamma, w\right)-V^{T}\left(w_{-(T-1)}, w\right) \geq V^{T}\left(w_{-(T-1)}, w\right)-V^{T}\left(w_{-(T-1)}-\gamma, w\right) .
$$

Weak loss priority can be interpreted as a insecurity analogue of weak uncertainty aversion in the context of individual choice under uncertainty. Figure 5 provides an example of the application of this axiom. The three wealth streams indicated differ in the earliest period (period -5 ) only. The uppermost stream starts at a net wealth level of 5 , the second at 3 and the third at 1 . Thus, the absolute value of the difference between the first and the second stream in this period is the same as the absolute difference between the second and the third stream (this difference is given by 2). The distinguishing feature between these differences is that the first represents a loss while the second is a gain with respect to the middle stream. Weak loss priority requires that the loss has a larger impact on insecurity than the (equal-sized) gain so that

$$
V^{5}(5,3,3,-1,0,2)-V^{5}(3,3,3,-1,0,2) \geq V^{5}(3,3,3,-1,0,2)-V^{5}(1,3,3,-1,0,2) .
$$




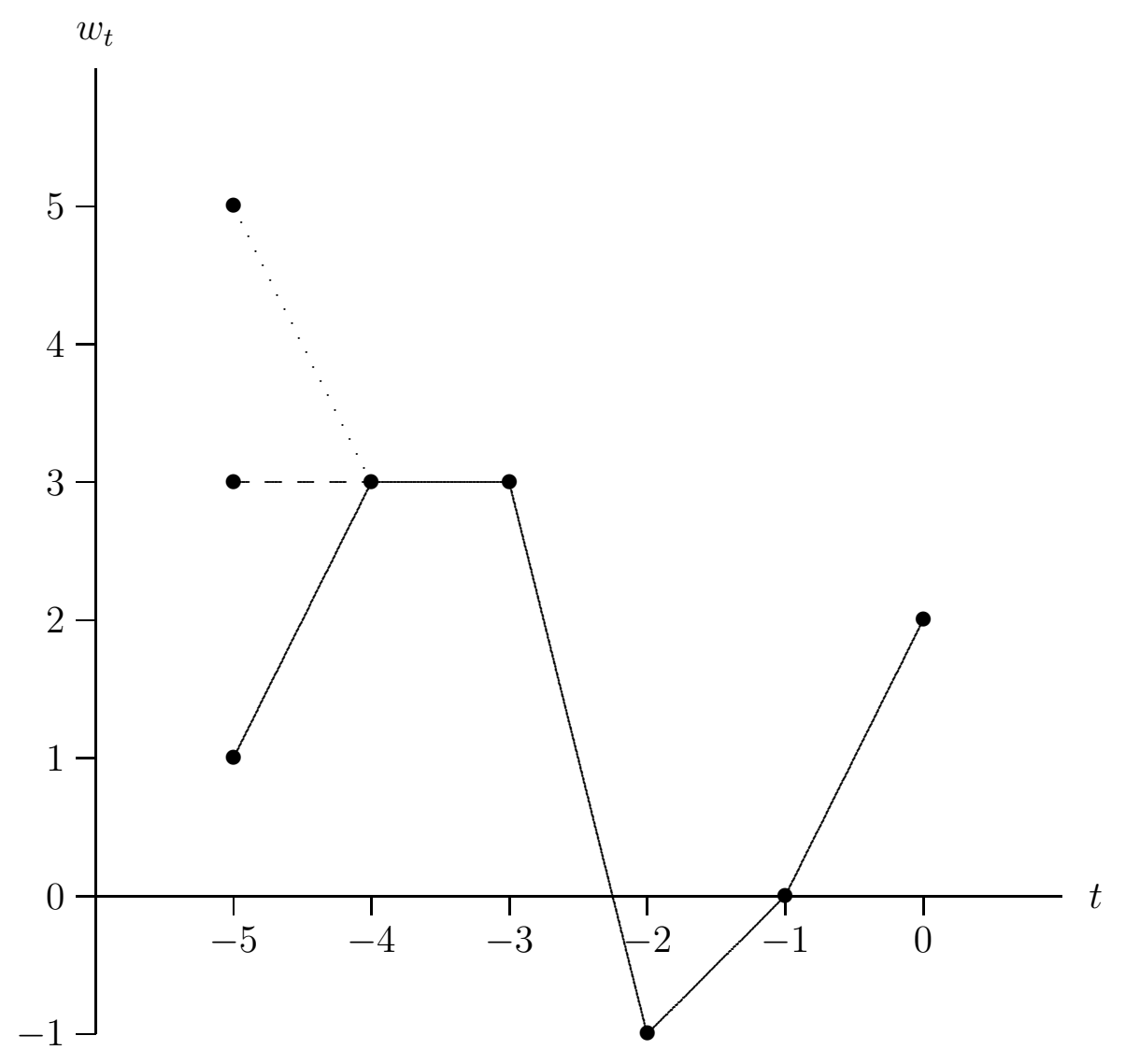

Figure 5: Weak Loss Priority.

Let $\mathcal{D}$ be the set of all pairs of sequences $(\alpha, \beta) \in \mathcal{C}^{2}$ such that

$$
\alpha_{-t} \geq \beta_{-t} \quad \text { for all } t \in \mathbb{N} \text {. }
$$

A two-sequences Gini insecurity measure $V_{(\alpha, \beta)}$ is rank-ordered if $(\alpha, \beta) \in \mathcal{D}$. Adding weak loss priority to the axioms of Theorem 1 leads to a characterization of the rank-ordered two-sequences Gini measures. That the rank-ordered two-sequences Gini measures satisfy weak loss priority is an immediate consequence of (26). The proof of the only-if part of the following theorem follows from Theorem 1 and the observation that weak loss priority implies (26), given that $V$ is a two-sequences Gini.

Theorem 2. A measure of individual insecurity $V$ satisfies difference monotonicity, the proximity property, homogeneity, translatability, the temporal aggregation property and weak loss priority if and only if there exists $(\alpha, \beta) \in \mathcal{D}$ such that $V=V_{(\alpha, \beta)}$.

Theorem 2 identifies the class of insecurity measures that we advocate in this paper. As an example, consider the measure obtained by choosing the sequences $\alpha$ and $\beta$ so that

$$
\alpha_{-t}=\frac{1}{2 t-1} \quad \text { and } \quad \beta_{-t}=\frac{\alpha_{-t}}{2}
$$


for all $t \in \mathbb{N}$. Clearly, $(\alpha, \beta) \in \mathcal{D}$. The coefficients according to the sequence $\alpha$ are the inverses of the coefficients corresponding to the Gini social-evaluation function; see, for instance, Donaldson and Weymark (1980) and Weymark (1981).

The extreme cases of the rank-ordered two-sequences Ginis in which $\beta_{-t}=0$ for all $t \in \mathbb{N}$ are not included in our theorems. This is the case because, due to their extreme nature (ignoring the effects of wealth gains altogether), these measures fail to satisfy the basic requirements of difference monotonicity and the proximity property. For that

reason, we do not state a formal characterization result but note that a characterization of these measures can be obtained if, in Theorem 1, the difference monotonicity property is amended so as to apply to losses only and ceteris paribus gains have to be assumed to leave insecurity unchanged and, in addition, the proximity property has to be reformulated so as to apply to losses only. Details are available from the authors on request.

\section{Concluding Remarks}

This paper suggests a rigorous and precise way of defining a measure of economic insecurity. We identify three fundamental properties any insecurity measure should possess. In addition, we axiomatize a class of measures with some intuitively appealing additional axioms and an important subclass.

Our measures are sufficiently flexible to admit the use of any model describing the formation of individual expectations. We intentionally do not pick a specific theory of decision-making under uncertainty in order to allow for a wide variety of possible applications. The exploration of such applications constitutes a natural next step in this area of research.

\section{References}

Blackorby, C., D. Primont and R.R. Russell (1978), Duality, Separability, and Functional Structure: Theory and Economic Applications, North-Holland, Amsterdam.

Bossert, W. (1990), "An Axiomatization of the Single-Series Ginis," Journal of Economic Theory, 50, 82-92.

Donaldson, D. and J.A. Weymark (1980), "A Single-Parameter Generalization of the Gini Indices of Inequality," Journal of Economic Theory, 22, 67-86. 
International Encyclopedia of the Social and Behavioral Sciences, 2001, Elsevier, Amsterdam.

Jacobs, E. (2007), "The Politics of Economic Insecurity," downloaded from http://www.brookings.edu/papers/2007/09politics_jacobs.aspx.

New Palgrave Dictionary of Economics, Second Edition, 2008, Palgrave Macmillan, Hampshire.

Osberg, L. (1998), "Economic Insecurity," Social Policy Research Centre, Discussion Paper No. 88.

Social Science Encyclopedia, Third Edition, 2008, Routledge, New York.

United Nations Department of Economic and Social Affairs (2008), "World Economic and Social Survey 2008. Overcoming Economic Insecurity," downloaded from http://www.un.org/esa/policy/wess/.

Weymark, J.A. (1981), "Generalized Gini Inequality Indices," Mathematical Social Sciences, 1, 409-430.

Zank, H. (2007), "Social welfare functions with a reference income," Social Choice and Welfare, 28, 609-636. 